\title{
Pulsatile ocular blood flow in subjects with sleep apnoea syndrome
}

Michal S. Nowak ${ }^{1}$, Piotr Jurowski ${ }^{1}$, Roman Gos ${ }^{1}$, Marek E. Prost ${ }^{2}$, Janusz Smigielski ${ }^{3}$

1Department of Ophthalmology and Visual Rehabilitation, Medical University of Lodz, Lodz, Poland

2Department of Ophthalmology, Military Institute of Aviation Medicine, Warsaw, Poland ${ }^{3}$ Department of Informatics and Medical Statistics, Medical University of Lodz, Poland

Submitted: 17 September 2009

Accepted: 25 November 2009

Arch Med Sci 2011: 7, 2: 332-336

DOI: 10.5114/aoms.2011.22087

Copyright (๑) 2011 Termedia \& Banach

\section{Abstract}

Introduction: The aim of the study was to determine the correlation between pulsatile ocular blood flow (POBF) and sleep apnoea syndrome (SAS).

Material and methods: Patients were recruited from those who underwent polysomnography in the "Sleep Unit" of the physiology department (Medical University, Lodz, Poland). A total of 52 Caucasian patients, 34 with SAS and 18 age- and gender-matched controls, were included in the study. Comprehensive ophthalmic examination included the pulsatile ocular blood flow (POBF) measurements, disc analysis with Heidelberg Retina Tomograph II and Oculus Centerfield computerized perimetry as well as the best visual acuity, a slit lamp and indirect ophthalmoscopic evaluation of anterior and posterior segments and applanation tonometry.

Results: The observed prevalence of glaucoma in SAS patients was $5.9 \%$ (2 of 34). The mean values of POBF were $1069.21 \pm 235.94 \mu \mathrm{l} / \mathrm{min}$ in the SAS group and $1061.78 \pm 174.63 \mu \mathrm{l} / \mathrm{min}$ in the control group. The study revealed that the differences of mean POBF between the SAS patients and the control group were not statistically significant: Mann-Whitney $U$-test $p>0.05$. No correlations were found between sleep apnoea syndrome and mean intraocular pressure (IOP), mean retinal nerve fibre layer (RNFL) thickness and visual field mean defect (MD).

Conclusions: No correlation was found between pulsatile ocular blood flow and sleep apnoea syndrome. Although some previous studies found an association between IOP, MD, RNFL thickness and sleep apnoea syndrome, our study did not confirm that. However, a high prevalence of glaucoma was found among SAS patients in Poland.

Key words: pulsatile ocular blood flow, sleep apnoea syndrome, glaucoma.

\section{Introduction}

Sleep apnoea syndrome (SAS) is a disease characterized by recurrent complete or partial upper airway obstruction during sleep. Inducing hypoxia, it may cause serious cardiovascular and neurological complications. The incidence of sleep apnoea is the highest in overweight and middle-aged or older men. Other risk factors associated with SAS include neck circumference, upper airway abnormalities, alcohol abuse and snoring. Several eye disorders have been found in association with SAS, including: floppy eyelid syndrome, keratoconus, optic neuropathy, non-arteritic anterior ischaemic optic neuropathy and papilloedema secondary to raised intracranial pressure [1-3].

\author{
Corresponding author: \\ Michal S. Nowak, MD, PhD \\ Department \\ of Ophthalmology \\ and Visual Rehabilitation \\ Medical University of Lodz \\ 113 Zeromskiego \\ 90-549 Lodz, Poland \\ Phone: +48 426393634 \\ Fax: +48 426393636 \\ E-mail: \\ michaelnovak@interia.pl
}


In 1999 Mojon et al. first found the association between SAS and either primary open angle glaucoma or normal tension glaucoma [4]. Since that time several reports have confirmed this association, and revealed that almost $20 \%$ of glaucoma patients present sleeping disorders. Recent studies have shown that there is also a correlation between SAS and either higher incidence of visual field defects or decrease in the retinal fibre layer [5-10]. Although, considering this evidence, the association between SAS and glaucoma may be suspected to be really strong, its mechanism still remains unknown or at least unclear.

There is increasing evidence that altered retinal blood flow is one of the major risk factors of developing glaucoma $[11,12]$. The major cause of this reduction in ocular blood flow is thought to be vascular dysregulation [11-14]. There is also evidence that pulsatile ocular blood flow (POBF) is significantly reduced in glaucoma patients $[15,16]$.

Pulsatile ocular blood flow is created by the pulsatile waveform as blood passes through the eye with each heartbeat. Calculation of the pulsatile component of the ocular blood flow is based on the amplitude and shape of the intraocular pressure (IOP) and on the heart rate. A built-in computer selects the five most representative pulses for the calculation of POBF ( $\mu \mathrm{l} / \mathrm{min})[17,18]$.

Pulsatile ocular blood flow measures the total ocular blood flow and it is known that choroidal circulation constitutes $85 \%$ of the total ocular blood flow. Since it is the choroidal flow, which supplies the optic nerve, POBF seems to be of importance in the evaluation of glaucomatous damage [19].

The aim of the study was to determine the correlation between POBF and sleep apnoea syndrome (SAS).

\section{Material and methods}

The study design was semi-prospective and cross-sectional. Patients were consecutively recruited from those who underwent polysomnography in the "Sleep Unit" of the physiology department (Medical University, Lodz, Poland). All of them were subjects of European Caucasian origin, most of whom live or lived in Poland. The diagnosis of sleep apnoea syndrome was based on whole night polysomnographic recordings. The apnoea-hypopnoea index was used to define the number of apnoeas and hypopnoeas experienced per hour of sleep. Sleep study findings for SAS were considered positive when the apnoea-hypopnoea index (AHI) was $\geq 5 / h$. Patients with sleep apnoea syndrome using a CPAP (continuous positive airway pressure) mask were excluded from the study. The controls were selected from those who had sleep studies for some indication but had $\mathrm{AHI}<5$. A total of 52 Caucasian patients, 34 with SAS and 18 ageand gender-matched controls, were included in the study. The study participants were contacted by telephone and scheduled for the ophthalmic examination from January 2006 to February 2008. The study adhered to the provisions of the Declaration of Helsinki for research involving human subjects and was approved by the institutional review board of the Medical University of Lodz, Poland.

\section{Ophthalmic examinations}

In each patient a single study eye was randomly chosen for the data. Comprehensive ophthalmic examination included the POBF measurements, disc analysis with Heidelberg Retina Tomograph II and Oculus Centerfield computerized perimetry as well as the best visual acuity, applanation tonometry, a slit lamp evaluation of the anterior segment and two $45^{\circ}$ fundus images of each selected eye. A complete medical history was taken for each patient. Pulsatile ocular blood flow was recorded in a sitting position after instillation of one drop of Alcaine. We used an ocular blood flow (OBF) system (supplied by OBF Laboratories UK, Ltd., Malmesbury, Wiltshire, UK) whose tonograph has been reported to have measurements of acceptable accuracy and reproducibility $[17,18]$. Other measurements with the OBF system included pulse amplitude, rate and volume. In this study, only mean POBF and IOP values were subjected to statistical analyses.

Computerized perimetry was performed by an experienced perimetrist using the glaucoma program Oculus Centerfield ver. 3.12r07 (supplied by Oculus Poland, Ltd., Warsaw, Poland), which is equivalent to 30.2 Humphrey visual field. Mean defect was calculated for both eyes in each patient. A visual field test was considered reliable if falsepositive and false-negative answers were $<15 \%$. Disc analysis with Heidelberg Retina Tomograph II (supplied by Heidelberg Engineering $\mathrm{GmbH}$, Heidelberg, Germany) included rim area, rim volume, linear cup/disc ratio and mean retinal nerve fibre layer (RNFL) thickness. For the diagnosis of primary open angle glaucoma (POAG) or ocular hypertension (OHT), the criteria based on the Ocular Hypertension Treatment Study had to be maintained [19].

\section{Data management and statistical analysis}

Data were entered into a Microsoft Excel database and all statistical analyses were performed using STATISTICA ver. 6.1 PL software (supplied by StatSoft Polska, Cracow, Poland). The correlation between $\mathrm{AHI}$ and age and body mass index (BMI) was examined with unpaired Student's 
t-test. Fisher-Behrens test was used to investigate the correlation between $\mathrm{AHI}$ and sex distribution as well as in diagnosis of systemic hypertension. Mean values of POBF, IOP and RNFL thickness were calculated. The differences between mean POBF, mean IOP, mean RNFL thickness in the sleep apnoea group and the controls were examined with Mann-Whitney $U$-test. All sample means are reported with their standard deviations. Differences were considered significant at $p<0.05$.

\section{Results}

\section{Study participants}

The demographic characteristics of the patients grouped according to $\mathrm{AHI}$ are presented in Table I. The study comprised 34 patients with SAS and 18 healthy controls. The mean value of AHI among sleep apnoea patients was $64.12 \pm 24.59$. Most of these patients were diagnosed with moderate to severe SAS. There was no significant difference between the groups in terms of sex distribution (Fisher-Behrens test, $p>0.05$ ). The mean age of the patient group was $51.4 \pm 9.2$ years, while in the control group it was $50.1 \pm 8.7$; there were no statistically significant differences in age distribution between the groups (unpaired Student's t-test, $t=0.35, p>0.05)$. The AHI was significantly associated with the body mass index (unpaired Student's $t$-test, $t=2.1, p<0.05$ ). But there was no correlation between $\mathrm{AHI}$ and systemic hypertension.
However, this finding was of borderline significance (Fisher-Behrens test, $p=0.06$ ).

\section{Ophthalmic examinations}

Reliable measurements were obtained in 52 eyes. Two patients were diagnosed with glaucoma. One had been previously diagnosed with POAG and treated with a topical $\beta$-blocker twice daily. Another patient had previously unrecognized normal tension glaucoma (NTG). Both of them had sleep apnoea syndrome. The observed prevalence of glaucoma in SAS patients was $5.9 \%$ (2 of 34). No OHT was found in the studied groups.

The results of POBF measurements in both groups are presented in Table II. The mean values of POBF were $1069.21 \pm 235.94 \mu \mathrm{l} / \mathrm{min}$ in the SAS group and $1061.78 \pm 174.63 \mu \mathrm{l} / \mathrm{min}$ in the control group. The study revealed that the differences in mean POBF were not statistically significant in the investigated groups: Mann-Whitney $U$-test $p>0.05$.

The results of IOP and RNFL thickness measurements are presented in Tables III-IV. No statistically significant differences in mean IOP and mean RNFL thickness were found in patients with sleep apnoea syndrome and in the controls: MannWhitney $U$-test $p>0.05$.

Computerized perimetry revealed defects in the visual field caused by glaucoma (POAG and NTG) in two patients. No correlation was found between the presence and absence of SAS and quantitative

Table I. Demographic data of the study participants

\begin{tabular}{|ccccc|}
\hline AHI & No. of patients & Age \pm SD [years] & Sex $(M / F)$ & $B M I \pm S D\left[\mathrm{~kg} / \mathrm{m}^{2}\right]$ \\
\hline$\geq 5$ & 34 & $51.4 \pm 9.2$ & $30 / 4$ & $31.5 \pm 4.7$ \\
\hline$<5$ & 18 & $50.1 \pm 8.7$ & $16 / 2$ & $28.3 \pm 4.0$ \\
\hline
\end{tabular}

Student's t-test: Age $t=0.35, p>0.05, B M I t=2.1, p<0.05$, Fisher-Behrens test: $\operatorname{sex} p>0.05$

$A H I$ - apnoea-hypopnoea index, BMI-body mass index

Table II. Characteristics of pulsatile ocular blood flow ( $\mu \mathrm{l} / \mathrm{min})$

\begin{tabular}{|lcccccc|}
\hline AHI & $n$ & Mean & Median & Min. & Max. & Std. dev. \\
\hline$\geq 5$ & 34 & 1069.21 & 1053.50 & 726.00 & 1674.00 & 235.94 \\
\hline$<5$ & 18 & 1061.78 & 1052.00 & 734.00 & 1378.00 & 174.63 \\
\hline All & 52 & 1066.64 & 1053.50 & 726.00 & 1674.00 & 214.94 \\
\hline
\end{tabular}

Mann-Whitney U-test $(p<0.05)$, POBF U $=297.0, Z=-0.164, p=0.870$

Table III. Characteristics of intraocular pressure $(\mathrm{mmHg})$

\begin{tabular}{|lllllll|}
\hline AHI & $n$ & Mean & Median & Min. & Max. & Std. dev. \\
\hline$\geq 5$ & 34 & 19.06 & 19.00 & 11.70 & 25.00 & 2.95 \\
\hline$<5$ & 18 & 18.71 & 17.70 & 15.90 & 22.50 & 2.52 \\
\hline All & 52 & 18.94 & 18.95 & 11.70 & 25.00 & 2.79 \\
\hline
\end{tabular}

Mann-Whitney U-test $(p<0.05), 10 P U=255.00, Z=0.979, p=0.328$ 
Table IV. Characteristics of retinal nerve fibre layer thickness $(\mathrm{mm})$ in HRT ॥

\begin{tabular}{|llllllc|}
\hline AHI & $n$ & Mean & Median & Min. & Max. & Std. dev. \\
\hline$\geq 5$ & 34 & 0.27 & 0.28 & 0.16 & 0.38 & 0.06 \\
\hline$<5$ & 18 & 0.24 & 0.21 & 0.14 & 0.37 & 0.07 \\
\hline All & 52 & 0.26 & 0.26 & 0.14 & 0.38 & 0.06 \\
\hline
\end{tabular}

Mann-Whitney U-test $(p<0.05), R N F L$ thickness $U=220.00, Z=1.646, p=0.099$

assessment of mean defect (MD). Other examinations, including the best visual acuity and evaluation of anterior and posterior segments, did not demonstrate any significant differences between the SAS group and the controls.

\section{Discussion}

In the present report the correlation of POBF and SAS was investigated. The study revealed that there was no correlation between mean pulsatile ocular blood flow measurements and sleep apnoea syndrome. Direct comparison of our results to the results obtained in other studies concerning POBF and glaucoma is limited due to the fact that those studies did not focus on patients with sleep apnoea syndrome. Previously published data showed that high-risk ocular hypertensive and primary open angle glaucoma patients with high IOP had significantly reduced pulsatile ocular blood flow when compared with low-risk hypertensive and healthy subjects $[15,16]$. The present study was inspired by an accumulating number of reports on the relation between altered ocular blood flow and the development of glaucoma [11-13, 20-22]. Due to the inaccessibility of the eye during sleep, we chose to study POBF in patients with sleeping disorders as we hypothesized that glaucomatous neuropathy in them may result from pathological ocular blood flow. However, according to Schmetterer et al. POBF might not be an adequate measure of total ocular blood flow. There are several limitations of POBF since the values are not obtained through direct measurement of ocular blood flow, but derived mathematically by estimating ocular pulse volume change based on preset equations relating ocular volume to IOP. This formula is based on the cardiac cycle and a standard scleral rigidity. Pulsatile ocular blood flow measurements are therefore affected by individual differences of scleral rigidity, ocular volume, heart rate, systemic blood pressure and IOP [17]. This may have biased the findings. Topical $\beta$-blocker in one glaucoma patient might also have interfered with ocular blood flow. However, in a recent study from Turkey concerning ocular blood flow and SAS, there was no statistically significant difference between ophthalmic artery resistivity index (OARI) and central retinal artery resistivity index (CRARI) between patients and controls $(p>0.05)$ [23]. Other hypotheses of the origin of glaucoma in SAS patients state that it may result from impaired optic nerve head blood flow autoregulation secondary to repetitive prolonged apnoeas. Alternatively, optic nerve vascular dysregulation might be secondary to SAS-induced arterial hypertension and arteriosclerosis or to the imbalance between nitric oxide (a vasodilator) and endothelin (a vasoconstrictor) [4]. What is interesting, in a study of POBF in healthy young adults, it was found that stimulated obstructive apnoeas, such as those generated by the Mueller manoeuvre, were associated with a concomitant increase in pulsatile ocular blood flow [24].

In our study we also investigated the correlations between the prevalence of glaucoma, IOP, RNFL thickness and visual field mean defect (MD) and SAS. The study revealed that the prevalence of glaucoma in our group of patients with sleep apnoea syndrome was $5.9 \%$ (2 of 34). Although the low number of patients in the present study was the most important limitation, our results were in agreement with the studies that support the association between SAS and glaucoma [4, 7, 8, 23].

In those reports that support this association, the prevalence of both primary open angle and normal tension glaucoma among the SAS population ranged from $5.9 \%$ to $12.9 \%[4,7,8,23]$. Contrary to these reports, Geyer et al. reported that the prevalence of glaucoma in SAS was similar to that in the general population [25]. Despite the fact that the link between SAS and glaucoma has been investigated with contradictory results, the prevalence of glaucoma among SAS patients in Poland was significantly higher than expected in a regular Caucasian population (1.7-3.0\%) [25-27].

Some previously published studies revealed that patients' respiratory disturbance index (RDI), i.e. the number of apnoeas experienced per hour, was positively correlated with IOP, visual field defects or decrease in the retinal fibre layer $[4,9,10,20]$. Contrary to the above reports, in our study we did not find any statistically significant association between mean IOP, mean RNFL thickness and MD with sleep apnoea syndrome. Although other studies had higher numbers of patients, our results were in agreement with Geyer et al., who published a cross-sectional report involving 228 participants with SAS and did not find a correlation between RDI 
and IOP. Demographic analysis of the participants of our study revealed that only the AHI was significantly associated with body mass index. The lack of significance for the association of SAS with systemic hypertension is most likely due to the small number of subjects.

In conclusion, in our group of patients there was no correlation between pulsatile ocular blood flow and sleep apnoea syndrome. Although some previous studies found an association between IOP, MD, RNFL thickness and sleep apnoea syndrome, our study did not confirm that. However, a high prevalence of glaucoma was found among SAS patients in Poland.

\section{Acknowledgments}

The abstract of this original paper was presented at the European Association for Vision and Eye Research Congress in Portoroz, Slovenia, on 1-4 October 2008.

\section{References}

1. McNab AA. The eye and the sleep. Sleep Med Rev 2007; 11: 269-76.

2. Dhillon S, Shapiro CM, Flanagan J. Sleep-disordered breathing and effects on ocular health. Can J Ophthalmol 2007; 42: 238-43.

3. Abdal H, Pizzimenti JJ, Purvis CC. The eye in sleep apnea syndrome. Sleep Med 2006; 7: 107-15

4. Mojon DS, Hess CW, Goldblum D, et al. High prevalence of glaucoma in patients with sleep apnea syndrome. Ophthalmology 1999; 106: 1009-12.

5. Mojon DS, Hess CW, Goldblum D, Böhnke M, Körner F, Mathis J. Primary open-angle glaucoma is associated with sleep apnea syndrome. Ophthalmologica 2000; 214: 115-8.

6. Onen SH, Mouriaux F, Berramdane L, Dascotte JC, Kulik JF, Rouland JF. High prevalence of sleep-disordered breathing in patients with primary open-angle glaucoma. Acta Ophthalmol Scand 2000; 78: 638-41.

7. Mojon DS, Hess CW, Goldblum D, et al. Normal tension glaucoma is associated with sleep apnea syndrome. Ophthalmologica 2002; 216: 180-4.

8. Sergi M, Salerno DE, Rizzi M, et al. Prevalence of normal tension glaucoma in obstructive sleep apnea syndrome patients. J Glaucoma 2007; 16: 42-6.

9. Tsang CS, Chong SL, Ho CK, Li MF. Moderate to severe obstructive sleep apnea patients is associated with higher incidence of visual fields defect. Eye 2006; 20: 38-42.

10. Kargi SH, Altin R, Koksal M, et al. Retinal fibre layer measurements are reduced in patients with obstructive sleep apnea syndrome. Eye 2005; 19: 575-9.

11. Grieshaber MC, Flammer J. Blood flow in glaucoma. Curr Opin Ophthalmol 2005; 16: 79-83.

12. Mozaffarieh M, Grieshaber MC, Flammer J. Oxygen and blood flow: players in the pathogenesis of glaucoma. Mol Vis 2008; 14: 224-33.

13. Fuchsjäger-Mayrl G, Wally B, Georgopoulos M, et al. Ocular blood flow and systemic blood pressure in patients with primary open-angle glaucoma and ocular hypertension. Invest Ophthalmol Vis Sci 2004; 45: 834-9.

14. Waliszek-Iwanicka A, Waliszek M, Banach M, Rysz J, Gos R. Assessment of blood flow in posterior ciliary arteries and its correlation with intraocular and arterial blood pressures in patients with open angle glaucoma. Med Sci Monit 2010; 16: CR501-9.

15. Agarwal HC, Gupta V, Sihota R, Singh K. Pulsatile ocular blood flow among normal subjects and patients with high tension glaucoma. Indian J Ophthalmol 2003; 51: 133-8.

16. Kerr J, Nelson P, O'Brien C. Pulsatile ocular blood flow in primary open-angle glaucoma and ocular hypertension. Am J Ophthalmol 2003; 136: 1106-13.

17. Schmetterer L, Dallinger S, Findl O, et al. Noninvasive investigations of the normal ocular circulation in humans. Invest Ophthalmol Vis Sci 1998; 39: 1210-20.

18. Yang YC, Hulbert MF, Batterbury M, Clearkin LG. Pulsatile ocular blood flow measurements in healthy eyes: reproducibility and reference values. J Glaucoma 1997; 6: 175-9.

19. Keltner JL, Johnson CA, Cello KE, et al. Classification of visual field abnormalities in the ocular hypertension treatment study. Arch Ophthalmol 2003; 121: 643-50.

20. Pemp B, Georgopoulos M, Vass C, et al. Diurnal fluctuation of ocular blood flow parameters in patients with primary open angle glaucoma and healthy subjects. Br J Ophthalmol 2009; 93: 486-91.

21. Tsai JC. Influencing ocular blood flow in glaucoma patients: the cardiovascular system and healthy lifestyle choices. Can J Ophthalmol 2008; 43: 347-50.

22. Nowak MS, Wybor K, Gos R, et al. Protective effect on visual functions of long-term use of trimetazidine in treatment of primary open angle glaucoma and degenerative myopia. Arch Med Sci 2007; 3: 152-6.

23. Karakucuk S, Goktas S, Aksu M, et al. Ocular blood flow in patients with obstructive sleep apnea syndrome (OSAS). Graefes Arch Clin Exp Ophthalmol 2008; 246: 129-34.

24. Lundmark PO, Trope GE, Flanagan JG. The effect of stimulated obstructive apnoea on intraocular pressure and pulsatile ocular blood flow in healthy young adults. Br J Ophthalmol 2003; 87: 1363-9.

25. Geyer O, Cohen N, Segev E, et al. The prevalence of glaucoma in patients with sleep apnea syndrome: same as in the general population. Am J Ophthalmol 2003; 136: 1093-6.

26. Mitchell P, Smith W, Attebo K, Healey PR. Prevalence of open-angle glaucoma in Australia. The Blue Mountains Eye Study. Ophthalmology 1996; 103: 1661-9.

27. Bron A, Baudouin C, Nordmann JP, et al. Prevalence of intraocular hypertension and glaucoma in a non-selected French population. J Fr Ophthalmol 2006; 29: 635-41. 